\title{
H-OBIS: A historical dimension to the ocean biogeographical information system
}

\author{
David J. Starkey \\ University of Hull • Hull UK \\ Poul Holm \\ University of Southern Denmark - Esbjerg Denmark \\ Tim Smith \\ North East Fisheries Science Center - Woods Hole, Massachusetts USA \\ Robert Francis \\ University of Washington • Seattle, Washington USA \\ Helen Rozwadowski \\ Georgia Institute of Technology • Atlanta, Georgia USA
}

C

ensuses offer snapshots of a given population at a particular point in time. In presenting a static picture of a dynamic process, such snapshots might reveal much about the composition and spatial distribution of the population in question, but they shed no light on its past development and are therefore unable to illuminate its likely future course. Without history, censuses lack a predictive quality. Recognizing this fact, the designers of the Census of Marine Life have incorporated an historical dimension into their scheme of study. This strand of the wider project, dubbed the 'History of Marine Animal Populations' (HMAP), will generate new, validated historical databases that will be fed into the Ocean Biogeographic Information System (OBIS). To test the feasibility of this process, it is intended that a pilot study (H-OBIS) will be conducted in 2001.

HMAP is being co-ordinated by a steering group comprised of Professor Poul Holm (University of Southern Denmark), Dr. Tim Smith (North East Fisheries Science Center, Wood's Hole, Mass), Dr. Robert Francis (University of Washington, Seattle) and Dr. David J. Starkey (University of Hull, UK). At a planning workshop held in Esbjerg, Denmark, in February 2000, thirty experts from over a dozen different countries discussed the aims, scope and methodology of HMAP (http://core.ssc.erc.msstate.edu/censhmap.html). Four basic research questions were defined:

- How has the extent and diversity of marine

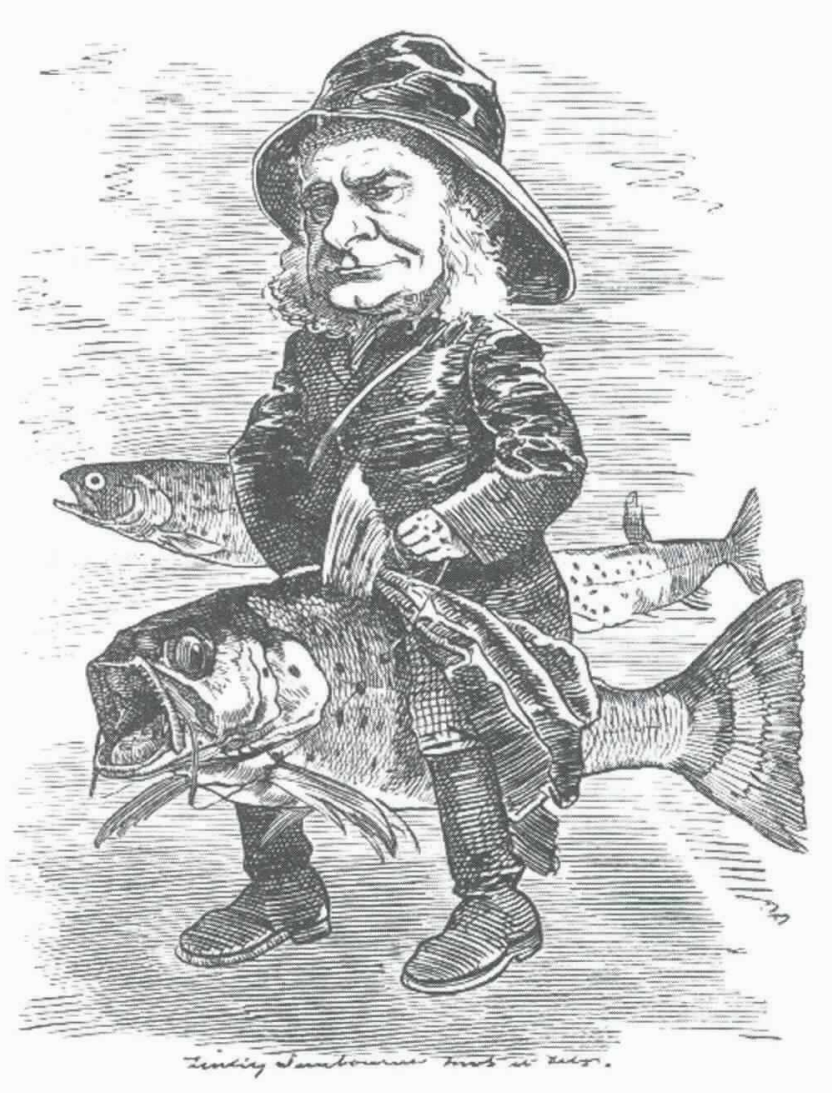

Punch, 18 March 1881. 
animal populations changed over the last 2,000 years?

- Which factors have forced or influenced the changing extent and diversity of marine animal populations?

- What has been the anthropogenic and biological significance of changes in marine animal populations?

- Has the interaction between marine ecosystems and human societies been significant?

To address these issues, HMAP will draw upon two distinct, but related disciplines. First, paleo-ecological methodologies will be employed to analyze natural (including sedimentary) archives in order to generate data that will underpin a reconstruction of the character and variability of a 'pristine' - i.e. before human harvesting commenced - marine environment. Second, more conventional historical techniques will be used to identify, validate and analyze documentary records relating to catches, catch effort and the sale of marine produce. Such information will be gleaned from a variety of primary sources generated for a variety of time- and place-contingent purposes. Accordingly, tax and tithe returns, state accounts, export data, log books and other documentary sources will be gutted to yield time series that will chart the impact of human exploitation and management of marine resources, and the role of such activity in the development of human societies. These methodologies will be applied in conjunction with environmental studies and modelling to establish how marine ecosystems have adapted to changing degrees of human intervention and variable natural influences.

The execution of this ambitious scheme poses problems of scale and data availability. To obviate these difficulties, HMAP will constitute a long-term research investigation into a select number, between 10 and 15, of the 50 marine ecosystems identified in one recent analysis. These case studies will be selected according to various criteria. While all will have experienced significant natural fluctuations and been subject to prolonged human exploitation, the quantity and quality of the sedentary and historical data available, the state of existing knowledge of the ecosystem, and the caliber of the personnel able and willing to undertake the research will also be taken into consideration. To inform this selection process, pilot studies will be undertaken.

Case study research teams will implement HMAP's research program. The teams will be multidisciplinary in character, with historians, paleo-ecologists and ecologists/biologists working together under the leadership of a Senior Researcher. To enhance the synergy of the research effort, the HMAP Steering Group will coordinate the work of the teams to facilitate crossregional comparative analyzes and to ensure methodological consistency. A multi-disciplinary approach will enable researchers to properly evaluate sources and data pertaining to the marine environment. Moreover, it will facilitate the construction and evaluation of long time-series of quantitative information regarding physical factors such as climate, currents, salinity, fish populations, etc., and human factors like catches, technology and demand patterns.

Such data will be generated to inform the Census of Marine Life (CoML) project. It will therefore be necessary to render it in such a way that it conforms to Census's database structure, the Ocean Biogeographic Information System (OBIS). This entails the translation of data derived from paleo-ecological and historical sources into standardized scientific datasets. One of the initial objectives of HMAP will be to conduct a pilot project (H-OBIS) to ensure that historical sources of information on marine animal populations can be successfully fitted into the categories dictated by the database structure. To avoid wasting time and money on the collection of virgin material for experimental purposes, this pilot project will use extant time-series to test the compatibility of historical data and OBIS. Directed by Dr. Helen Rozwadowski (Georgia Institute of Technology), the pilot project will assess the utility for CoML purposes of the evidence assembled by Randy Reeves on North Atlantic humpback whales and Pacific bowhead and grey whales. This substantial stock of information has been taken from the logbooks of the whaling ships that worked in both the Atlantic and the Pacific during the nineteenth and early twentieth centuries. Reeves has successfully drawn on this data to offer preliminary conclusions about changes in the size and spatial distribution of the whale populations, as well as shifts in their breeding grounds. These findings will be integrated with the global whale catch data series generated for the twentieth century by a team led by Cherry Allison (International Whaling Commission, Cambridge, UK). The past will thereby be connected to the present day.

The H-OBIS pilot study will provide a model for the integration of the statistical outcomes of the HMAP case studies into the OBIS framework. If this can be accomplished, a dynamic, human element will be instilled into the overarching Census project. Furthermore, the vision of a Census of Marine Life that draws upon the past to help explain the present and predict the future will be brought a step closer to realization. 